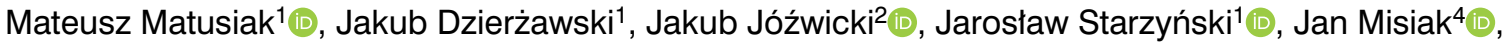
Anna A. Brożyna ${ }^{1,3}$ (D) Wojciech Jóźwicki1 ${ }^{1,4}$

${ }^{1}$ Department of Tumor Pathology and Pathomorphology, Oncology Centre-Prof. Franciszek Łukaszczyk Memorial Hospital, Bydgoszcz, Poland

2Department of Clinical Pathomorphology, Faculty of Medicine, Ludwik Rydygier Collegium Medicum in Bydgoszcz, Nicolaus Copernicus University in Torun, Bydgoszcz, Poland ${ }^{3}$ Department of Human Biology, Faculty of Biology and Environment Protection, Nicolaus Copernicus University, 87-100 Toruń, Poland; ${ }^{4}$ Department of Tumor Pathology and Pathomorphology, Nicolaus Copernicus University Collegium Medicum in Bydgoszcz, Bydgoszcz, Poland

Mateusz Matusiak and Jakub Dzierżawski are equal first authors

\title{
Expression of PD-L1 in tumor and immune system cells affects the survival of patients with urinary bladder cancer
}

\section{Corresponding author:}

Wojciech Jóźwicki, Department of Tumor Pathology and Pathomorphology, Oncology Centre-Prof. Franciszek Łukaszczyk Memorial Hospital, Bydgoszcz 85-796,

Romanowskiej 2 St., Poland, e-mail: jozwickiw@co.bydgoszcz.pl

Medical Research Journal 2019; Volume 4, Number 3, 142-147 10.5603/MRJ.a2019.0026 Copyright (C) 2019 Via Medica ISSN 2451-2591

\begin{abstract}
Background: The prediction of tumor malignancy is still one of the most demanding diagnostic tasks in urinary bladder cancer because of its clinicopathological heterogeneity. The aim of this study was to evaluate the expression of PD-L1 in tumor cells (TCS) and immune effector cells (IECs) as well as the pattern of distribution of PD-L1 + IECS within the tumor (dispersed or aggregated) and their association with survival of patients with pT1-pT4 urinary bladder cancer.

Materials and methods: 110 patients with stage pT1-pT4 urothelial bladder carcinoma who underwent radical cystectomy/cystoprostatectomy between 2011 and 2014 were included in the study. Paraffin blocks most representative of the tumor were selected for H\&E staining as well as immunostaining with the use of rabbit anti-PD-L1 (Ventana clone SP142, Roche). In each sample, the area of the tumor containing PDL1+ IECs, as well as, the pattern of distribution (dispersed or aggregated) of PD-L1+ immune effector cells within the tumor were analyzed. In addition, the expression of PD-L1 in TCs was also assessed. Results: Patients had a shorter survival time in pT2-pT4 cases without TCs expressing PD-L1 $(p=0.007)$ and/or when PD-L1 + IECs displayed a predominantly dispersed pattern of distribution ( $p=0.013$ ).

Conclusions: The expression of PD-L1 on TCs and IECs is a prognostic factor which allows for stratification of patient survival in UBC. The predominance of dispersed or aggregated pattern of distribution of PD-L1 + IECs in the tumor may be considered as a new prognostic factor in PT1-T4 UBC and indicate the functional status of the immune system.

Key words: PD-L1; urothelial bladder cancer; tumor microenvironment; immune cell distribution, immune effector cells, immune checkpoint inhibitors
\end{abstract}

Med Res J 2019; 4 (3): 142-147

\section{Introduction}

Urothelial bladder cancer (UBC) is the seventh most frequency occurring cancers in men and may be responsible for 200000 deaths in 2018 [1]. The assessment of the tumor malignancy requires the assessment of tumor advancement (pT), histological malignancy $(\mathrm{G})$, number of nonclassic differentiation types (NDN) and the tissue invasion type (TIT) [2-4]. Unfortunately, the prediction of the tumor progression and the risk of recurrence remains a diagnostic and therapeutic challenge in UBC. Its histological and clinical heterogeneity which likely reflects its molecular heterogeneity makes understanding the biological mechanisms of UBC malignancy a challenging area of research [5-8]. Recent studies have indicated several probable mechanisms of tumor progression by the way of modulating the immune system anti-tumor response. One important mechanism is the suppression of anti-tumor response by an excess infiltration of regulatory $\mathrm{T}$ cells (Tregs) [9]. The over-expression of RCAS1 in tumor cells (TCs) and the surrounding cells of the cancer microenvironment were also shown to correlate with clinical and pathomorphological patterns of malignancy [5]. An 
interesting new area of research is the cancer immune evasion through activation of immune checkpoints which suppress IECs $[10,11]$. One such mechanism is the PD-1/PD-L1 signalling pathway which suppresses the activity of $T$ lymphocytes particularly in the effector phase of the immune response [11]. In 2017, Xingyuan et al. performed studies that showed the ability of UBC TCs to induce immunosuppression in patients through upregulation of PD-L1 expression on tumor-associated macrophages (TAMs) with the involvement of IL-10 [12]. Furthermore, PD-L1 can be expressed in both TCs and IECs. There is evidence that its expression within the tumor may in some circumstances facilitate the escape from immune surveillance and lead to tumor progression [10,13]. The results of clinical trials have demonstrated a significant correlation between PDL1 expression in some tumors and the effectiveness of treatment using immune checkpoint inhibitors (ICl) [14-19], especially in early stages of the disease [20], and in combination therapies [21]. However, some patients who meet the criteria for therapy do not respond well to anti-PD-L1 treatment [22, 23]. Thus, finding new eligibility criteria for immunotherapy to increase its efficacy and safety is paramount. The aim of this study is to assess the expression of PD-L1 on TCs and IECs which are present in the tumor area and to analyze the effect of PD-L1 expression on patient survival in UBC.

\section{Materials and methods}

A total of 110 patients with pT1-pT4 UBC from Oncology Centre Prof. Franciszek Łukaszczyk Memorial Hospital in Bydgoszcz (Poland) who underwent radical cystectomy (or cystoprostatectomy) in 2011-2014 were enrolled in this study. The average follow-up time after surgery was about 22 months. The clinico-pathological characteristics of the study group is presented in Table 1.

Staging and histological type of tumors were assessed according to the WHO classification [24]. Survival data were collected from the National Health Fund. The study was approved by the Committee of Ethics of Scientific Research of Collegium Medicum, Nicolaus Copernicus University, Poland (KB 587/2018).

\section{The preparation and evaluation of H\&E stained samples}

Tissue sections were fixed in $10 \%$ buffered formalin and embedded in paraffin blocks according to a standard protocol. The evaluation of HE (Hematoxylin-Eosin) stained sections from each urinary bladder was performed and one representative paraffin-embedded block was chosen for immunohistochemical staining.
Table 1. Patient clinico-pathological characteristics

\begin{tabular}{|c|c|}
\hline Patient's characteristics & Number of patients \\
\hline \multicolumn{2}{|l|}{ Age (years) } \\
\hline $30-50$ & 2 \\
\hline $51-60$ & 24 \\
\hline $61-70$ & 53 \\
\hline $71-80$ & 29 \\
\hline $81-90$ & 2 \\
\hline \multicolumn{2}{|l|}{ Sex } \\
\hline Female & 22 \\
\hline Male & 88 \\
\hline \multicolumn{2}{|l|}{ pT } \\
\hline 1 & 12 \\
\hline $2 a$ & 13 \\
\hline $2 b$ & 12 \\
\hline $3 a$ & 10 \\
\hline $3 b$ & 31 \\
\hline $4 a$ & 32 \\
\hline $4 b$ & 0 \\
\hline \multicolumn{2}{|l|}{$\mathrm{pN}$} \\
\hline Not available & 2 \\
\hline 0 & 61 \\
\hline 1 & 12 \\
\hline 2 & 33 \\
\hline 3 & 2 \\
\hline \multicolumn{2}{|l|}{ Recurrence } \\
\hline Yes & 16 \\
\hline No & 94 \\
\hline
\end{tabular}

\section{Immunohistochemical staining and evaluation of samples}

Immunohistochemical staining of $4 \mu \mathrm{m}$ sections was performed with rabbit monoclonal anti-PD-L1 (Ventana clone SP142, Roche) and visualization system OptiView DAB IHC Detection Kit and OptiViewAmplification Kit using VENTANA BenchMark system, according to the manufacturer's protocol. To confirm the specificity of the signal, the same protocol but without the use of anti-PD-L1 was performed as a negative control for each sample. For each staining cycle, a positive control sample of human tonsil was included following the manufacturer's recommendations. In each test sample, the percentage of tumor area occupied by tumor-associated immune cells exhibiting PD-L1 positive staining was assessed, regardless of staining intensity or the number of ICs present [25] (Fig. 1A, B). Furthermore, the pattern of distribution of PD-L1 + IECs in the tumor was evaluated as either dispersed or aggregated (with cell aggregates of 10 cells or more in a high-power field of view) (Fig. 1C, D). 

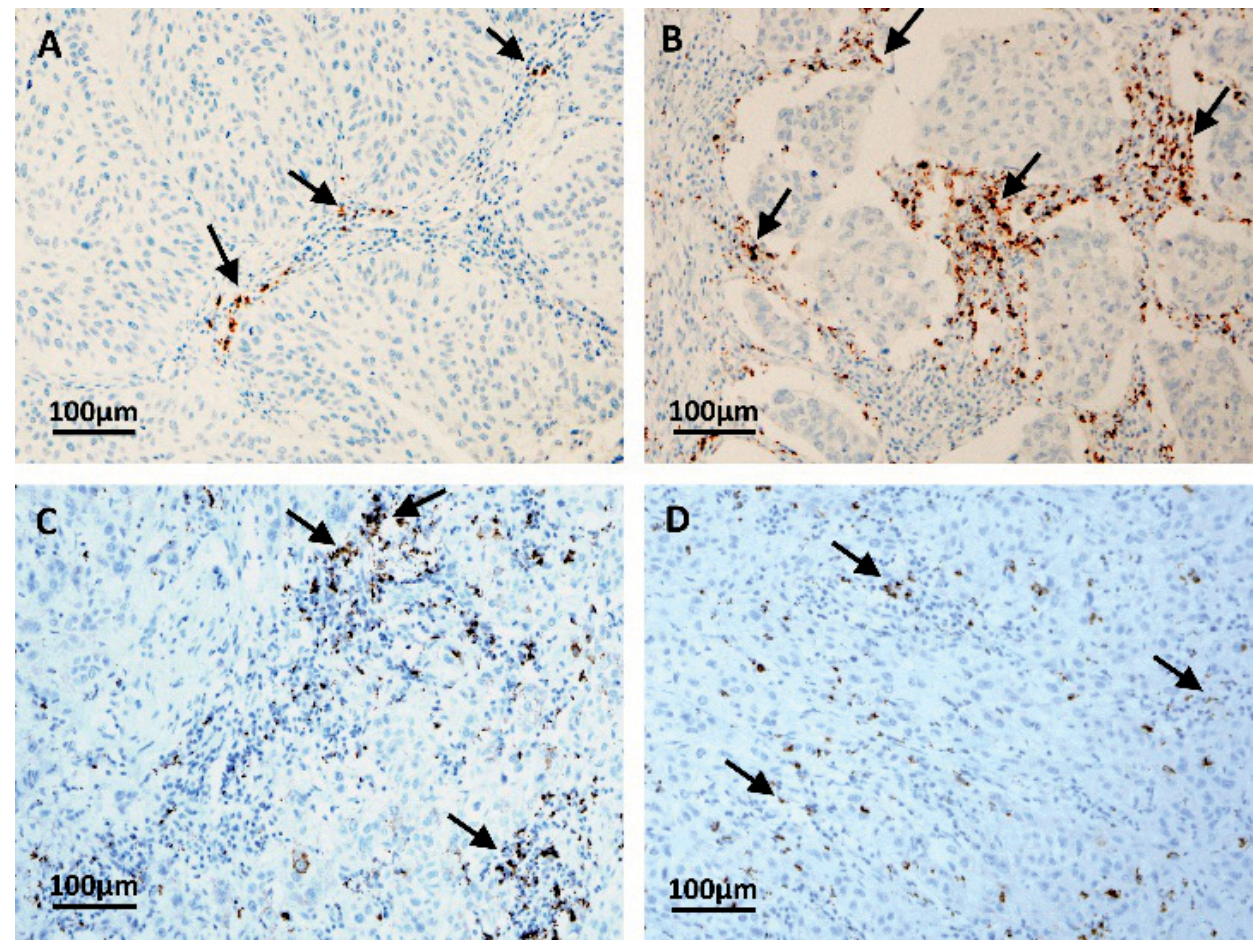

Figure 1. The panel gives an example of the extent of the presence of PD-L1+ IECs in the tumor: $<5 \%(A)$ and $\geq 5 \%(B)$. The aggregated and dispersed presence of PD-L1+ IECs is shown in figures C and D, respectively. Arrows point to immune cells
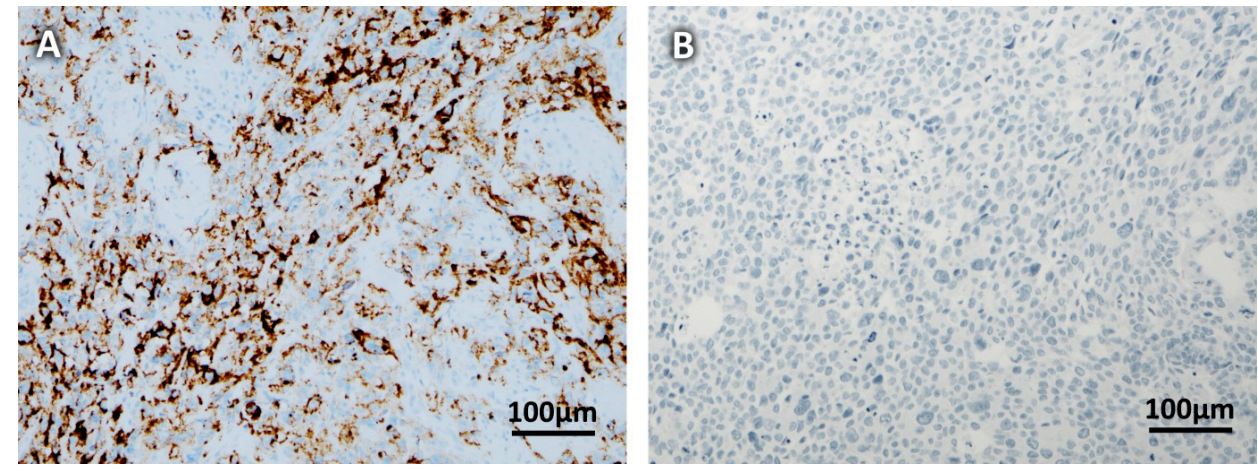

Figure 2. The picture shows sample images of the presence of PD-L1 expression in tumor cells (A) and its absence (B)

The presence or absence of PD-L1 expression was evaluated in the TCs regardless of the intensity and extent of the expression.

The microscopic assessment was performed with the use of Nikon Eclipse 80i microscope. Pictures were taken with Nikon Digital Sight DS Fi1-U2 camera and with NISElements BR 3.0 software (Nikon Instruments Europe B.V., Badhoevedorp, The Netherlands).

\section{Statistical analysis}

The relationship between PD-L1 expression in TCs and IECs was analyzed using T-test for independent sam- ples. PD-L1 expression in TCs and IECs and the probability of survival was assessed with Kaplan-Meier curves. The statistical analyses were performed using STATISTICA data analysis software (version 8.0; StatSoft, Inc., Tulsa, OK, USA). A p-value $<0.05$ was considered to be significant.

\section{Results}

\section{Expression of PD-L1 and survival}

The analysis of PD-L1 expression in TCs showed that the probability of survival was higher in cases where 


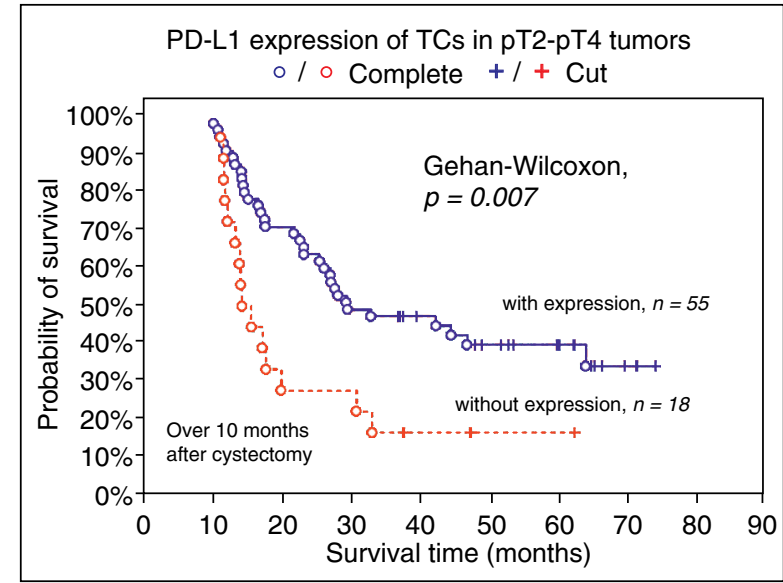

Figure 3. The survival probability 20 and 50 months after cystectomy for UBC was about two and a half times and twice higher, respectively, in patients with tumor PD-L1 expression

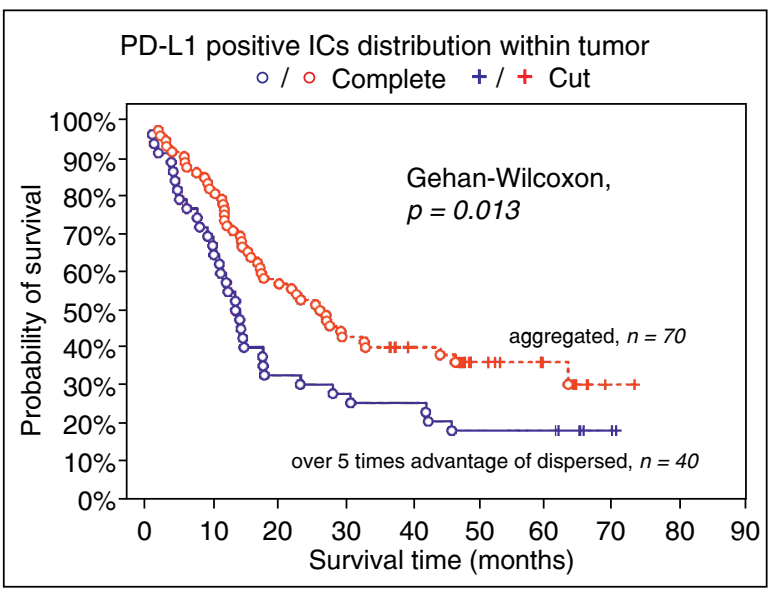

Figure 4. The probability of survival 4 years after cystectomy for UBC was more than twice lower in patients with the advantage of dispersed PD-L1 + IECs in tumor

TCs expressed PD-L1 compared to those where such expression was not found (Fig. 3).

Further, we observed that the presence of PD-L1+ IECs with a dispersed distribution pattern $(n=40)$ was associated with significantly lower survival probability (Fig. 4).

\section{Expression of PDL1 in tumor and immune cells}

We observed a strong correlation between the extent of PD-L1 expression in TCs and the extent of expression of PD-L1 in IECs in close vicinity $(p<0.05 ; r=0.60$; $n=110$ ). The extent of PD-L1 expression in TCs was particularly high in tumors where PD-L1+ IECs occupied more than $5 \%$ of the whole tumor area (Fig. 5).

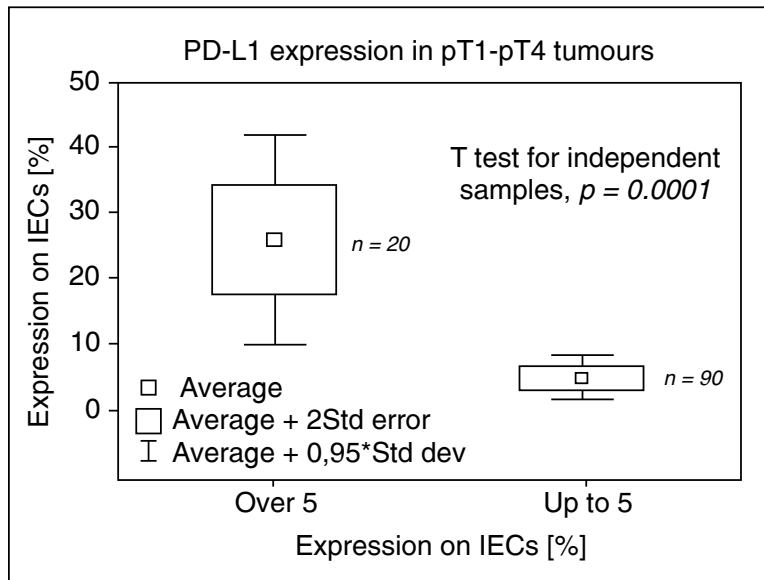

Figure 5. The average extent of PD-L1 expression in tumor cells is at least 5 times as high in tumors in which PD$\mathrm{L} 1+$ immune cells occupy over $5 \%$ of their surface area

\section{Discussion}

Immune mechanisms play a key role in tumor development and progression. Inhibition of anti-tumour response of the immune system and induction of immune tolerance are observed in the early and late stage of UBC development, respectively $[5,9$, 26-28]. Recent studies have focused on immune checkpoint modulation of IECs activity by means of a PD1-PDL1 signalling pathway in UBC [11, 29]. We have demonstrated that the expression of PD-L1 in TCs of UBC is associated with a higher probability of survival (Fig. 3). Contrary findings were obtained by Chun-Te et al. and also by Yide et al. [30, 31]. However, the results of several other authors are not in accord. Bellmunt et al. and Davickai et al. confirmed such relation in patients with UBC [32, 33], likewise, Kim et al. detected an association in head and neck tumors [34]. We cannot rule out that the difference in findings may be due to an adopted selection of samples (i.e. non-epithelial bladder cancer included) and due to differences in methodology (a type of antibody, representative section sampling). Some other authors report that the expression of PD-L1 on TCs may be associated with the increase of PD-L1 + IECs numbers within the tumor [31]. In our study, we have observed that the increase of the extent of the tumor with PD-L1 + IECs is associated with the increase of PD-L1 expression in TCs (Fig. 5). This may suggest a similar or even synergistic involvement of these two cellular components (TCs and IECs) in downregulation of anti-tumor response through PD-L1. There is no clear consensus on that matter in literature $[35,36]$. The studies on the role of PD-L1 molecule in ovarian cancer demonstrated that PD-L1 expression in tumor infiltrating macrophages 
may confer them protection from the immune system. The macrophages remove debris of tumor cells which in effect leads to high levels of tumor antigens in them which may result in them being targeted by IECs. PDL1 expression on the surface of macrophages may protect them against this autoimmune response [37, 38]. Thus, a parallel increase of PD-L1 expression in immune and tumor cells suggests a mechanism in which the immune system tries to activate a protective mechanism for its own effector cells. Ottenhof et al. in their study on the prognostic value of PD-L1 expression in penile cancer discovered an association between the dispersed pattern of ligand expression in tumor cells and a lower probability of survival [39]. In our study, the probability of survival in patients with UBC in stages pT1-T4 was reduced twice with a dispersed distribution of PD-L1+ IECs (Fig. 4). The findings we obtained suggest that the effectiveness of immune checkpoints linked to PD-L1 expression on IECs dispersed within the tumor is higher in comparison with PD-L1 located on aggregated IECs in UBC. Pichler et al. concluded that outcome prediction in UBC is most effective when the expression of PD-L1 on IECs is assessed. Their studies indicated, however, that high heterogeneity of expression dependent on the type of cells expressing PD-L1 cells may lead to unreliable results [40]. Recent studies suggest that not only the level of PD-L1 expression in IECs may have a prognostic value but also their location and aggregation within the tumor may significantly affect survival $[41,42]$. Our findings indicate that there is a significant prognostic value of assessing the dispersed and aggregated patterns of distribution of PD-L1+ IECs within the tumor. The diagnostic assessment standards for UBC proposed by other authors do not include the assessment of the distribution of PD-L1 + IECs within the tumor [25]. In our opinion, dispersion or aggregation of immunocompetent cells may be an indication of the status of the immune system and as such should be included in the eligibility criteria for immunotherapy in UBC.

\section{Conclusions}

The assessment of PD-L1 expression in TCs allows for prognostic stratification of patients with UBC in all stages. The predominance of dispersed or aggregated type of distribution of PD-L1 + IECs in the tumor may be considered as a new prognostic factor in pT1-pT4 UBC and may also indicate the status of the immune system of a patient. The assessment of the PD-L1 expression in TCs and IECs as well as of the type of distribution of IECs PD-L1 + in the tumor should be considered for inclusion in immunotherapy eligibility criteria in patients with UBC.

\section{Grant support}

This study was supported in part by funds for statutory research from Collegium Medicum, Nicolaus Copernicus University.

\section{Conflicts of interest}

The authors declare no conflicts of interest.

\section{List of abbreviations}

IECs - immune effector cells

TCs - tumor cells

PD-L1 - programmed death ligand 1

PD-1 - programmed death 1

$\mathrm{ICI}$ - immune checkpoint inhibitors

UBC - urothelial bladder cancer

\section{References}

1. Bray F, Ferlay J, Soerjomataram I, et al. Global cancer statistics 2018: GLOBOCAN estimates of incidence and mortality worldwide for 36 cancers in 185 countries. CA Cancer J Clin. 2018; 68(6): 394-424, doi: 10.3322/caac.21492, indexed in Pubmed: 30207593.

2. Jozwicki W, Domaniewski J, Skok Z, et al. Usefulness of histologic homogeneity estimation of muscle-invasive urinary bladder cancer in an individual prognosis: a mapping study. Urology. 2005; 66(5): 1122-1126, doi: 10.1016/j.urology.2005.06.134, indexed in Pubmed: 16286151.

3. Domanowska E, Jozwicki W, Domaniewski J, et al. Muscle-invasive urothelial cell carcinoma of the human bladder: multidirectional differentiation and ability to metastasize. Hum Pathol. 2007; 38(5): 741-746, doi: 10.1016/j.humpath.2006.11.001, indexed in Pubmed: 17306328.

4. Bellmunt J, Orsola A, Leow JJ, et al. ESMO Guidelines Working Group. Bladder cancer: ESMO Practice Guidelines for diagnosis, treatment and follow-up. Ann Oncol. 2014; 25 Suppl 3: iii40-iii48, doi: 10.1093/annonc/mdu223, indexed in Pubmed: 25096609.

5. Jóźwicki W, Brożyna AA, Siekiera J, et al. Expression of RCAS1 correlates with urothelial bladder cancer malignancy. Int J Mol Sci. 2015; 16(2): 3783-3803, doi: 10.3390/ijms16023783, indexed in Pubmed: 25674852.

6. Jóźwicki W, Skok Z, Brożyna A, et al. Urological Oncology Prognostic and diagnostic implications of histological differentiation in invasive urothelial cell carcinoma of the bladder: variant or non-classic differentiation number. Central European Journal of Urology. 2010; 63: 112-116, doi: 10.5173/ceju.2010.03.art1.

7. Jóźwicki W, Brożyna AA, Siekiera J. Expression of OCT4A: the first step to the next stage of urothelial bladder cancer progression. Int J Mol Sci. 2014; 15(9): 16069-16082, doi: 10.3390/ijms150916069, indexed in Pubmed: 25216339.

8. Amin MB. Histological variants of urothelial carcinoma: diagnostic, therapeutic and prognostic implications. Mod Pathol. 2009; 22 Suppl 2: S96-S9S118, doi: 10.1038/modpathol.2009.26, indexed in Pubmed: 19494856.

9. Jóźwicki W, Brożyna AA, Siekiera J, et al. Frequency of CD4+CD25+Foxp3+ cells in peripheral blood in relation to urinary bladder cancer malignancy indicators before and after surgical removal. Oncotarget. 2016; 7(10): 11450-11462, doi: 10.18632/oncotarget.7199, indexed in Pubmed: 26862849.

10. Sharma P, Allison JP. The future of immune checkpoint therapy. Science. 2015; 348(6230): 56-61, doi: 10.1126/science.aaa8172, indexed in Pubmed: 25838373

11. Okazaki T, Honjo T. PD-1 and PD-1 ligands: from discovery to clinical application. Int Immunol. 2007; 19(7): 813-824, doi: 10.1093/intimm/dxm057, indexed in Pubmed: 17606980. 
12. Wang X, Ni S, Chen Q, et al. Bladder cancer cells induce immunosuppression of $T$ cells by supporting PD-L1 expression in tumour macrophages partially through interleukin 10. Cell Biol Int. 2017; 41(2): 177-186, doi: 10.1002/cbin 10716, indexed in Pubmed: 27987237.

13. Freeman GJ, Long AJ, Iwai $Y$, et al. Engagement of the PD-1 immunoinhibitory receptor by a novel $B 7$ family member leads to negative regulation of lymphocyte activation. J Exp Med. 2000; 192(7): 1027-1034, doi: 10.1084/jem 192 7.1027, indexed in Pubmed: 11015443.

14. Rosenberg JE, Hoffman-Censits J, Powles T, et al. Atezolizumab in patients with locally advanced and metastatic urothelial carcinoma who have progressed following treatment with platinum-based chemotherapy: a single-arm, multicentre, phase 2 trial. Lancet. 2016 387(10031): 1909-1920, doi: 10.1016/S0140-6736(16)00561-4, indexed in Pubmed: 26952546.

15. Powles T, Eder JP, Fine GD, et al. MPDL3280A (anti-PD-L1) treatment leads to clinical activity in metastatic bladder cancer. Nature. 2014 515(7528): 558-562, doi: 10.1038/nature13904, indexed in Pubmed: 25428503

16. Ohaegbulam KC, Assal A, Lazar-Molnar E, et al. Human cancer immunotherapy with antibodies to the PD-1 and PD-L1 pathway. Trends Mol Med. 2015; 21(1): 24-33, doi: 10.1016/i.molmed.2014.10.009, indexed in Pubmed: 25440090.

17. Bellmunt J, de Wit R, Vaughn DJ, et al. KEYNOTE-045 Investigators. Pembrolizumab as Second-Line Therapy for Advanced Urothelial Carcinoma. N Engl J Med. 2017; 376(11): 1015-1026, doi: 10.1056/NEJMoa1613683, indexed in Pubmed: 28212060

18. Plimack ER, Bellmunt J, Gupta S, et al. Safety and activity of pembrolizumab in patients with locally advanced or metastatic urothelial cancer (KEYNOTE-012): a non-randomised, open-label, phase 1b study. Lancet Oncol. 2017; 18(2): 212-220, doi: 10.1016/S14702045(17)30007-4, indexed in Pubmed: 28081914

19. Zhang X, Shi X, Li J, et al. PD-1 Blockade Overcomes Adaptive Immune Resistance in Treatment with Anchored-GM-CSF Bladder Cancer Cells Vaccine. J Cancer. 2018; 9(23): 4374-4381, doi: 10.7150/jca.25423, indexed in Pubmed: 30519342.

20. Markowitz GJ, Havel LS, Crowley MJp, et al. Immune reprogramming via PD-1 inhibition enhances early-stage lung cancer survival. $\mathrm{JCl}$ Insight. 2018; 3(13), doi: 10.1172/jci.insight.96836, indexed in Pubmed: 29997286.

21. Szabados B, van Dijk N, Tang YZ, et al. Response Rate to Chemotherapy After Immune Checkpoint Inhibition in Metastatic Urothelial Cancer. Eur Urol. 2018; 73(2): 149-152, doi: 10.1016/j.eururo.2017.08.022, indexed in Pubmed: 28917596.

22. de Jong JJ, Stoop H, Nieboer D, et al. Concordance of PD-L1 expression in matched urothelial bladder cancer specimens. Histopathology. 2018; 73(6): 983-989, doi: 10.1111/his.13710, indexed in Pubmed: 30003574

23. He J, Hu Y, Hu M, et al. Development of PD-1/PD-L1 Pathway in Tumor Immune Microenvironment and Treatment for Non-Small Cell Lung Cancer. Sci Rep. 2015; 5: 13110, doi: 10.1038/srep13110, indexed in Pubmed: 26279307.

24. Sobin LH, Gospodarowicz MK, Wittekind CT. Classification of Malignant Tumours - 7th ed. Chichester. ; 2009: 262-5.

25. Jóźwicki W. VENTANA PD-L1 (SP142) - principles of pathomorphological evaluation in urinary bladder cancer. ; 2017.

26. Jóźwicki W, Brożyna AA, Siekiera J, et al. Changes in Immunogenicity during the Development of Urinary Bladder Cancer: A Preliminary Study. Int J Mol Sci. 2016; 17(3): 285, doi: 10.3390/ijms17030285, indexed in Pubmed: 26927070.

27. Chen $\mathrm{T}$, Wang $\mathrm{H}$, Zhang $\mathrm{Z}$, et al. A novel cellular senescence gene SENEX, is involved in peripheral regulatory $T$ cells accumulation in aged urinary bladder cancer. PLoS One. 2014; 9(2): e87774, doi: 10.1371/journal.pone.0087774, indexed in Pubmed: 24505313.

28. Darrasse-Jèze G, Bergot AS, Durgeau A, et al. Tumor emergence is sensed by self-specific CD44hi memory Tregs that create a dominant tolerogenic environment for tumors in mice. J Clin Invest. 2009; 119(9): 2648-2662, doi: 10.1172/JCl36628, indexed in Pubmed: 19652360.

29. Tzeng A, Diaz-Montero CM, Rayman PA, et al. Immunological Correlates of Response to Immune Checkpoint Inhibitors in Metastatic Urothelial Carcinoma. Target Oncol. 2018; 13(5): 599-609, doi: 10.1007/s11523-018-0595-9, indexed in Pubmed: 30267200.

30. Wu CT, Chen WC, Chang YH, et al. The role of PD-L1 in the radiation response and clinical outcome for bladder cancer. Sci Rep. 2016; 6: 19740, doi: 10.1038/srep19740, indexed in Pubmed: 26804478.

31. Huang $Y$, Zhang SD, McCrudden $C$, et al. The prognostic significance of PD-L1 in bladder cancer. Oncol Rep. 2015; 33(6): 3075-3084, doi: 10.3892/or.2015.3933, indexed in Pubmed: 25963805.

32. Mullane SA, Werner L, Rosenberg J, et al. Association of PD-L1 expression on tumor-infiltrating mononuclear cells and overall survival in patients with urothelial carcinoma. Ann Oncol. 2015; 26(4): 812-817, doi: 10.1093/annonc/mdv009, indexed in Pubmed: 25600565

33. Davick J J Frierson HF, Smolkin M et al. PD-L1 expression in tumor cells and the immunologic milieu of bladder carcinomas: a pathologic review of 165 cases. Hum Pathol. 2018; 81: 184-191, doi: 10.1016/j. humpath.2018.06.028, indexed in Pubmed: 29969606.

34. Kim HR, Ha SJ, Hong MH, et al. PD-L1 expression on immune cells, but not on tumor cells, is a favorable prognostic factor for head and neck cancer patients. Sci Rep. 2016; 6: 36956, doi: 10.1038/srep36956, indexed in Pubmed: 27841362

35. Faraj SF, Munari E, Guner G, et al. Assessment of tumoral PD-L1 expression and intratumoral CD8 + T cells in urothelial carcinoma. Urology. 2015; 85(3): 703.e1-703.e6, doi: 10.1016/j.urology.2014.10.020, indexed in Pubmed: 25733301

36. Mukherji D, Jabbour MN, Saroufim M, et al. Programmed Death-Ligand 1 Expression in Muscle-Invasive Bladder Cancer Cystectomy Specimens and Lymph Node Metastasis: A Reliable Treatment Selection Biomarker? Clin Genitourin Cancer. 2016; 14(2): 183-187, doi: 10.1016/j.clgc.2015.12.002, indexed in Pubmed: 26775720

37. Webb JR, Milne K, Kroeger DR, et al. PD-L1 expression is associated with tumor-infiltrating $T$ cells and favorable prognosis in high-grade serous ovarian cancer. Gynecol Oncol. 2016; 141(2): 293-302, doi: 10.1016/j.ygyno.2016.03.008, indexed in Pubmed: 26972336.

38. Webb JR, Milne K, Nelson BH. PD-1 and CD103 Are Widely Coexpressed on Prognostically Favorable Intraepithelial CD8 T Cells in Human Ovarian Cancer. Cancer Immunol Res. 2015; 3(8): 926-935, doi: 10.1158/2326-6066.CIR-14-0239, indexed in Pubmed: 25957117.

39. Ottenhof SR, Djajadiningrat RS, de Jong J, et al. Expression of Programmed Death Ligand 1 in Penile Cancer is of Prognostic Value and Associated with HPV Status. J Urol. 2017; 197(3 Pt 1): 690-697, doi: 10.1016/j.juro.2016.09.088, indexed in Pubmed: 27697578.

40. Pichler R, Fritz J, Lackner F, et al. Prognostic Value of Testing PD-L1 Expression After Radical Cystectomy in High-risk Patients. Clin Genitourin Cancer. 2018; 16(5): e1015-e1024, doi: 10.1016/j.clgc.2018.05.015, indexed in Pubmed: 29960831

41. Wang Bo, Pan W, Yang M, et al. Programmed death ligand- 1 is associated with tumor infiltrating lymphocytes and poorer survival in urothelial cell carcinoma of the bladder. Cancer Sci. 2019; 110(2): 489-498, doi: 10.1111/cas.13887, indexed in Pubmed: 30548363.

42. Jiang $Y$, Lo AWI, Wong A, et al. Prognostic significance of tumor-infiltrating immune cells and PD-L1 expression in esophageal squamous cell carcinoma. Oncotarget. 2017; 8(18): 30175-30189, doi: 10.18632/oncotarget.15621, indexed in Pubmed: 28404915. 